\title{
Introducing a Stabilizer Formulation for Allergenic Mold Extracts
}

\author{
Nazila Ariaee ${ }^{1}$, Mojtaba Sankian $^{2}$, AbdolrezaVarasteh $^{1}$, \\ Malihe Moghadam², Farahzad Jabbari*1
}

\begin{abstract}
Background: Sensitization to common mold allergens is one of the major causes of allergic rhinitis and asthma. Therefore, there is a critical need for standard sensitivity tests including skin prick tests to improve the stability of fungi extracts in traditional allergenic formulations. To address this concern, the present study aimed to develop a formulation to preserve allergenic activity of mold extracts.

Methods: 48 stabilizer formulations were designed and monitored for allergenic activity during a 40-days incubation period at $37^{\circ} \mathrm{C}$ using an ELISA. Specifically, the IgE reactivity of allergenic A. alternata extracts were examined. After establishing the most effective stabilizer formulation, we evaluated whether it could protect the allergenic activity of Alt a1, A. fumigatus, and C. herbarum using an IgE inhibition ELISA after 40 days at $37^{\circ} \mathrm{C}$.

Results: We demonstrated that the most effective stabilizer formulation was a glycerol-based extract containing Arg and Glu. This formulation had an equal ratio of sucrose, sorbitol and protein and was able to preserve more than $95 \%$ of allergenic A. alternata extract activity during a 40-days incubation period at $37^{\circ} \mathrm{C}$.

Conclusions: The present study reveals a novel formulation that is an efficient stabilizer of allergenic mold extract activity and has practical applications in mold skin prick tests, ELISAs, immunotherapies, and RAST.
\end{abstract}

Keywords: Allergy, Allergic Rhinitis, Mold, Stabilization, Skin Prick test.

\section{Introduction}

Allergic rhinitis is a common, chronic, inflammatory disease that is estimated to affect about $30 \%$ of the population worldwide (1). Allergic rhinitis places a huge burden on the health care system annually and affects the quality of life of those affected (2). Therefore, there has been increased attention and effort towards improving traditional diagnostic tests for allergens of interest and to minimize the risk of allergy development (3).

Many investigations have shown that molds are one of the main causes of allergenic minitis (4). Through the inhalation of mold spores, the immune system of sensitive patients triggers the onset of common allergy related symptoms including itchy and watery eyes, a runny nose, nasal congestion, and sneezing (5). In addition to atopy, fungi can cause a plethora of diseases including superficial skin lesions, asthma, fatal systemic mycoses, amongst other infectious diseases $(6,7)$. In this regard, mold extracts have critical industrial and clinical applications (8).

In the past decade, numerous studies have been working to develop allergenic mold extracts for skin prick testing, immunotherapies, enzyme-linked immunosorbent assays (ELISA), and radioallergosorbent testing (RAST) (9). Currently, there is a major concern on how to stabilize mold allergens within allergenic mold extracts (10). Since allergenic mold extracts have high protease content, stabilizing their allergenic activity has proven to be more challenging compared to other allergenic extracts. Importantly, industrial guidelines require allergenic extracts to remain active for two years during storage conditions at $4{ }^{\circ} \mathrm{C}(11)$.

Often, current formulations being used in clinical practice for skin prick testing and immunotherapy are complex matrices of proteins and glycoproteins. 
However, only a small portion of these extracts are active mold allergens, and the presence of other allergenic ingredients can cause significant hindrances to their reactivity during sensitivity testing (12). Therefore, the present study was conducted to design an effective stabilizer formulation to improve and maintain mold allergenic extract activity, while keeping in line with industrial demands.

\section{Materials and methods}

A total of 45 participants, from the allergy clinic of Qaem hospital in Mashhad Iran, who were between the ages of 15 and 55, were enrolled in the study. This study included patients with allergic rhinitis and thino conjunctivitis. 15 sensitive patients were selected for each common allergenic mold including Alternaria alternata (A. alternata), Aspergillus fumigatus (A. fumigatus), and Cladosporium herbarum (C. herbarum) (13). Sensitivity was proven by a positive skin prick test for the allergenic mold (Stallergern Greer, USA). This study was granted approval by the Ethics Committee of Mashhad University of Medical Sciences (code: 940043).

\section{Allergenic Extracts}

Standard strains of A. alternata (PTCC5224), A. fumigatus (PTCC 5009), and $C$. herbarum (PTCC5202) were obtained from the Iranian Research Organization for Science and Technology, and grown at $24{ }^{\circ} \mathrm{C}$ for 20 days on Sabouraud dextrose broth. The cultures followed a $12 \mathrm{~h}$ night and $12 \mathrm{~h}$ day schedule. A high allergenic extract yield was determined by the presence of both spores and mycelia using light microscopy.

Crude A. alternata was harvested and resuspended at a 1:10 ratio (w/v) in a $50 \mathrm{mM}$ carbonate buffer $(\mathrm{pH}$ 7.5). The buffer contained $5 \mathrm{mMol}$ ethylenediaminetetraacetic acid (EDTA), $1 \mathrm{mMol}$ phenylmethylsulfonylfluoride (PMSF), $5 \mathrm{mMol}$ thiourea, and of $2 \%$ insoluble polyvinylpyrrolidone (PVP). Following an overnight incubation at $4{ }^{\circ} \mathrm{C}$, the crude mold product was removed by filtration. The filtrate was then placed in 5\% cold acetone solution, which resulted in the formation of a precipitate. Through centrifugation, the acetone was removed, and the precipitate subsequently was dissolved in $25 \mathrm{mMol}$ Tris-HCl buffer ( $\mathrm{pH} 7.5$ ) containing $1 \mathrm{mg}$ per $\mathrm{ml} \mathrm{3-[(3-}$ Cholamidopropyl) dimethyl ammonio]-1- propanesulfonate (CHAPS). The resulting solution was dialyzed for a molecular cutoff of $12 \mathrm{kDa}$ against a potassium phosphate buffer.

\section{Setting the Stabilizer Formula}

To evaluate the effect of sugar on A. alternata extract stability, sorbitol and sucrose were included in the final formulation proportionally to the protein concentration, which was measured using a Bradford assay. The first group had an equal ratio of protein, sorbitol, and sucrose (1:1:1), whereas, the second group had the same amount of protein and sucrose but reduced amount of sorbitol (1:1:0.25). Further, the control group did not contain any sugar or polyol. Moreover, $150 \mathrm{mM}$ phosphate buffer saline (PBS) was used as the final solvent buffer for all extracts. In addition to sugar, $25 \mathrm{mM}$ glutamate (Glu) and arginine (Arg), and $1 \% \beta$-Alanine ( $\beta$-Ala) (14), glycerol $(20 \%)$, and human serum albumin (h Alb) $(0.03 \%)$ were also critically evaluated for their ability to stabilize allergenic $A$. alternata extracts. In total, there were 16 reactions between the chosen amino acids and $\mathrm{h}$ Alb (Table 1), and 48 stabilizers, which were categorized into three main groups based on the ratio of sugar to protein.

\section{Evaluating allergenic activity via ELISA}

We evaluated the efficacy of sugar, amino acids, $h$ $\mathrm{Alb}$, and glycerol on allergenic A. alternata extract stability using an ELISA and during an incubation period of 28 days at $37^{\circ} \mathrm{C}$. The incubation time was pre-selected according to the Arrhenius equation, and is equivalent to two years if incubated at $4{ }^{\circ} \mathrm{C}$ (15). Plates pre-coated with treated extracts (100 $\mu \mathrm{g} / \mathrm{ml}$ ) in $200 \mathrm{mM}$ bicarbonate buffer ( $\mathrm{pH}$ 9.6) were incubated overnight at $37{ }^{\circ} \mathrm{C}$, and subsequently blocked and incubated at $37{ }^{\circ} \mathrm{C}$ for $1 \mathrm{~h}$ the following day with $2 \%$ bovine serum albumin (BSA) in PBS buffer. Then, approximately $100 \mu \mathrm{l}$ of pooled sera, collected from 15 Alternaria sensitive patients, were added to designated wells. Bound IgE in pooled patient sera were detected after consecutive incubations with biotinylated Anti-human IgE (Santa Cruz), streptavidin-horseradish peroxidase (HRP) (Becton Dickinson, USA) and 3,3',5,5'Tetramethylbenzidine (TMB). Absorbance values obtained from a spectrophotometer at a wavelength of $540 \mathrm{~nm}$ were used to quantify concentration. 


\section{IgE inhibition ELISA}

Due to obtaining ambiguous data from our previous ELISA, we alternatively used a specific IgE inhibition ELISA and increased the incubation period from 28 to 40 days. We first coated plates with fresh $A$. alternata mold extract without further treatment. Then, we incubated pooled patient sera with mold extracts with different stabilizers at RT for $2 \mathrm{~h}$. Within the patient sera, specific IgE was inhibited by the allergenic activity of mold extracts, and a low absorbance value was indicative of high allergenic activity.

Negative control solutions were prepared either without any extract or antibody, or with substrate only. The negative control of inhibition prepared by mixing pooled sera with PBS, PBS replaced by extract to have no inhibition. Positive control solutions were prepared by incubating pooled sera with fresh extract, and represented as a percentage. This percentage reflects the allergenic activity of treated allergenic $A$. alternata extracts with specific IgE.

\section{Evaluating Alternaria allergenic activity}

The concentrations of Alt al (major allergen of $A$. alternata) were determined using a commercial sandwich ELISA kit (Indoor Biotechnologies, UK) that was performed in accordance to the manufacturer's instructions and recommendations.

\section{Results}

\section{Stabilizers effect on IgE reactivity of the extract}

As previously described, we conducted an ELISA to examine the effect of possible stabilizer candidates on IgE reactivity (see methods). Through the ELISA, we were able to detect $A$. alternata allergen-specific IgE in pooled patient sera after 28 days of incubation at $37^{\circ} \mathrm{C}$. Firstly, we determined the effect of sugar on $A$. alternata extract stability. The group with an equal ratio of sucrose, sorbitol, and protein had a significantly higher reactivity to $A$. alternata allergen-specific $\mathrm{IgE}$ in pooled patient sera compared to other groups reaching an average of $73.7 \pm 3.5$. The average $\mathrm{IgE}$ reactivity in the $A$. alternata extract with $25 \%$ sorbitol and no sugar group was $50.7 \pm 4.9$ and $48.8 \pm 4.9$ respectively, however, there was no significant difference between these two groups.

Secondly, we evaluated the stabilizing effect of amino acids, glycerol and $\mathrm{h}$ Alb. Our data revealed that the addition of Arg or Glu alone significantly improved allergenic $A$. alternata extract activity. However, the simultaneous addition of Arg and Glu was more effective and improved the activity up to $75 \pm 2.8$ while non-treated controls reached only $30 \pm 12.7$. Further, comparing the effect of $\beta$-Ala to Arg or Glu, revealed that there was no significant difference in allergenic activity between these three amino acids. However, comparing Arg/Glu to $\beta$-Ala treatment revealed a significantly higher level of $\mathrm{IgE}$ reactivity with Arg/Glu treatment. Lastly, we found that both $\mathrm{h} \mathrm{Alb}$ and glycerol did not preserve A. alternata extract allergenic potency.

In addition to using these stabilizers separately (Table 1), they were also used in combination with each other (Fig. 1). We showed that the group with equal sucrose, sorbitol, and protein, combined with Arg/Glu/glycerol (98\%), was the best at preserving allergenic activity. there was no significant difference between this treatment Arg/Glu/glycerol and the fresh extract. Further, there was no significant difference in allergenic activity between the reduced sorbitol group with $\mathrm{Arg} / \mathrm{Glu} / \mathrm{B}$ Ala (91\%) and Arg/Glu/glycerol (94\%), which was also similar to our positive control. Lastly, we found that the no sugar group, combined with $\mathrm{Arg} / \mathrm{Glu} / \mathrm{glycerol}$ had an allergenic activity (90\%) similar to our fresh extract \%. Overall, we found that the presence of amino acids, $\mathrm{h}$ Alb, and glycerol alone or in combination of each other had no effect on $A$. alternata extract stability, as there was no significant difference in allergenic activity after a 28-day incubation period at $37^{\circ} \mathrm{C}$ compared to controls.

Table 1. Stabilizers formulations based on amino acids groups. These groups are the same in main three groups regarding sugars.

\begin{tabular}{|c|c|}
\hline \multirow{3}{*}{$\begin{array}{l}\text { Arg } \\
(25 \mathrm{mM})\end{array}$} & $\operatorname{Albh}(0.03 \%)$ \\
\hline & Glycerol (20\%) \\
\hline & $\beta \mathrm{Ala}(2 \%)$ \\
\hline \multirow{3}{*}{$\begin{array}{l}\text { Glu } \\
(25 \mathrm{mM})\end{array}$} & $\operatorname{Albh}(0.03 \%)$ \\
\hline & Glycerol (20\%) \\
\hline & $\beta \mathrm{Ala}(2 \%)$ \\
\hline \multirow{3}{*}{$\begin{array}{l}\text { Arg/Glu } \\
(50 \mathrm{mM})\end{array}$} & $\operatorname{Albh}(0.03 \%)$ \\
\hline & Glycerol (20\%) \\
\hline & $\beta \operatorname{Ala}(2 \mathrm{v} \%)$ \\
\hline \multirow{3}{*}{$\begin{array}{l}\text { Without } \\
\text { amino acids }\end{array}$} & $\operatorname{Albh}(0.03 \%)$ \\
\hline & Glycerol (20\%) \\
\hline & $\beta$ Ala $(1 \%)$ \\
\hline
\end{tabular}




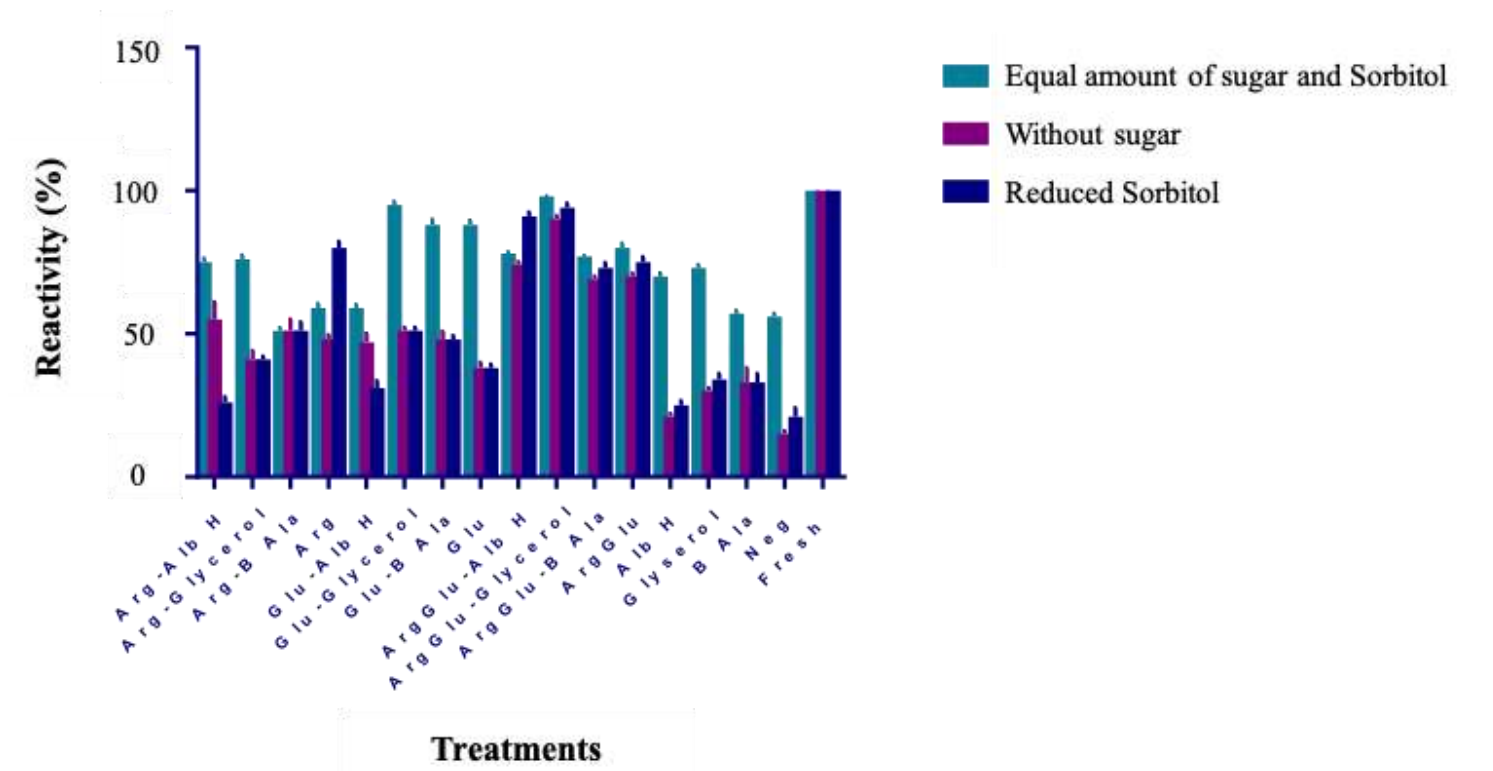

Fig. 1. Allergenic $A$. alternata extract activity after a 28 -day incubation period at $37^{\circ} \mathrm{C}$ with different stabilizers.

To achieve an effective stabilizer formulation, the allergenic potency of $A$. alternata extracts investigated using a specific IgE inhibition ELISA during an incubation period of 40 days at $37^{\circ} \mathrm{C}$. Our results show that the group with an equal sorbitol, sucrose and protein ratio had a higher percentage of specific $\mathrm{IgE}$ inhibition (Fig. 2). The average IgE inhibition among this group was $82 \pm 2.4$, which was significantly higher compared to the no sugar and reduced sorbitol group. The average specific IgE inhibition among $A$. alternata extracts without any sugar was $62 \pm 3.2$. In contrast, the group with reduced sorbitol had an average specific IgE inhibition of $64 \pm 2.3$. In conclusion, we found that there was no significant difference in specific IgE inhibition between the no sugar and reduced sorbitol groups.

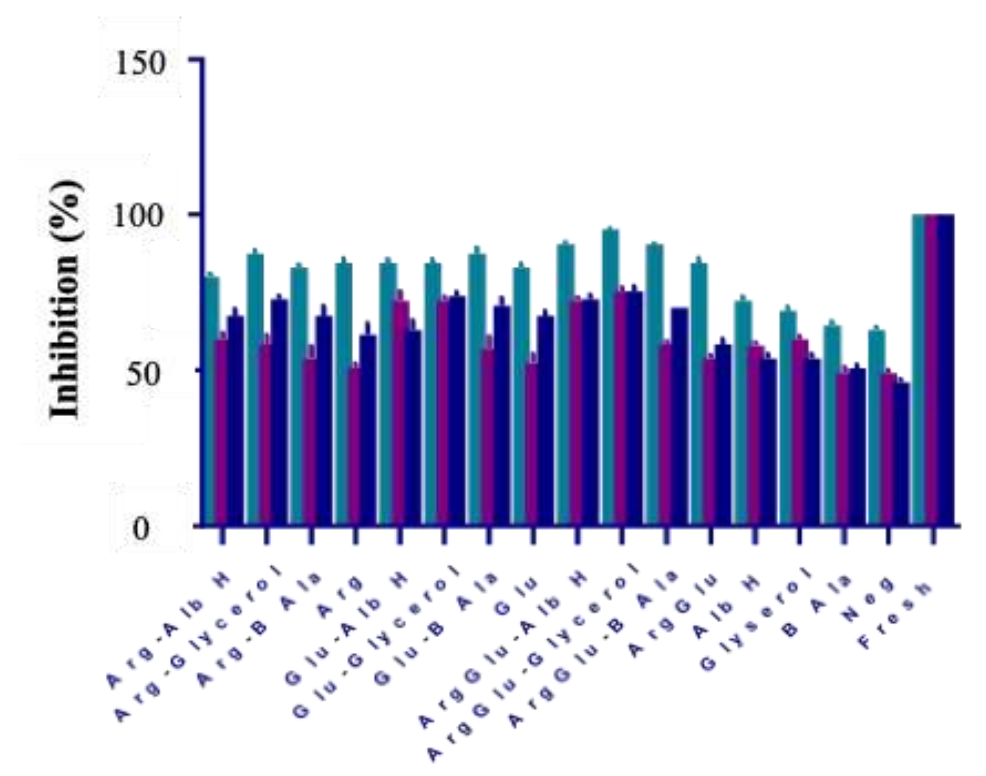

Equal amount of sugar and Sorbitol

Without sugar

Reduced Sorbitol

\section{Treatments}

Fig. 2. Inhibition of specific IgE in pooled patient sera with different allergenic A. altemata extract formulations aftera 40 -day incubation period at $37^{\circ} \mathrm{C}$. 
Further, specific IgE inhibition in the presence of Arg, Glu, Arg/Glu, $\beta$-Ala, h Alb, and glycerol was evaluated. We found that the addition of these stabilizers had no profound effect on allergenic $A$. alternata extract activity compared to untreated controls.

We then evaluated the combinatorial effect of these stabilizers, since there was no significant impact on allergenic $A$. alternata extract activity on their own.
The formulation containing an equal sorbitol, sucrose and protein ratio and $\mathrm{Arg} / \mathrm{Glu} / \mathrm{Glycerol}$ reached a specific IgE inhibition of $95 \% \pm 0.05$. In the same sugar group, combining $\mathrm{Arg} / \mathrm{Glu} / \mathrm{\beta}-\mathrm{Ala}$ or $\mathrm{Arg} / \mathrm{Glu} / \mathrm{h}$ Alb reached a specific IgE inhibition of $90.7 \%$. To conclude, we used the most effective stabilizer formulation, that protected more than $95 \%$ of $A$. alternata extract activity, to evaluate the activities of Alt al and other allergenic mold extracts (Table 2).

Table 2. Selected formulation ingredient.

\begin{tabular}{cccccc}
\hline $\begin{array}{c}\text { Protein content } \\
(\mathbf{m g} / \mathbf{m l})\end{array}$ & $\begin{array}{c}\text { Solvent buffer } \\
(\mathbf{m M})\end{array}$ & Sugar $(\mu \mathrm{g} / \mathrm{ml})$ & Glycerol (\%) & h Albumin & $\begin{array}{c}\text { Amino acids } \\
(\mathbf{m M})\end{array}$ \\
\hline 100 & PBS (150) & $\begin{array}{l}\text { Sorbitol }(100) \\
\text { Sucrose }(100)\end{array}$ & 20 & - & $\operatorname{Arg}(25)$ \\
& & & & Glu (25) \\
\hline
\end{tabular}

\section{Altal activity}

We conducted an Alt a1 ELISA on days 7, 14, 21, 28, and 40 to compare Alt al levels in the presence of our selected formulation and untreated extract, or untreated fresh extract. Further, we evaluated Alt al levels in the presence of a formulation similar to our selected formulae. Our data showed that during a 40-day incubation period, there was decreased Alt al activity $(43 \%)$ in untreated extracts. In comparison to the

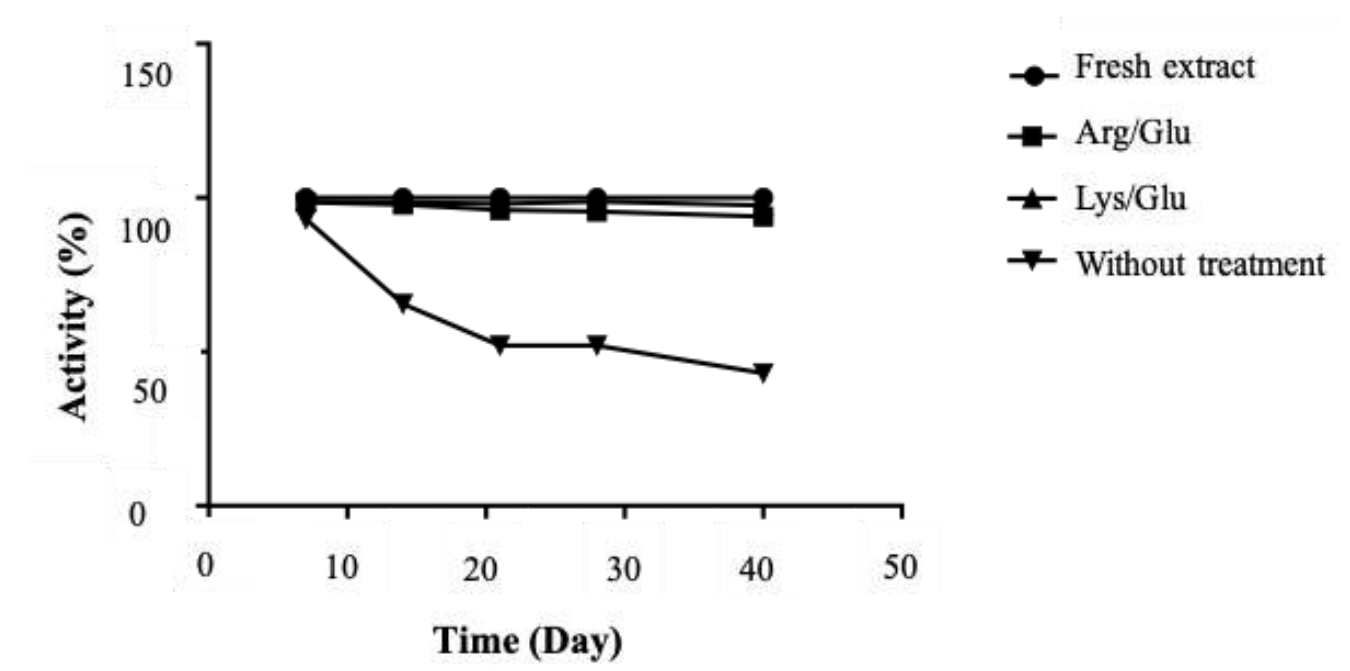

untreated extract, our selected stabilizer formulation had a significant impact on Alt al activity. While the activity of the fresh extract was normalized to $100 \%$, our selected stabilizer formulation showed minimal decline in Alt al activity and reached $97.5 \%$ after 40 days. Moreover, replacing Arg with Lys demonstrated similar results (93.9\%), and there was no significant difference compared to our selected formulation (Fig. 3).

Fig. 3. Alt al activity during 40 days of incubation at $37^{\circ} \mathrm{C}$ while treated with different stabilizers.

\section{Other molds}

The potency of allergenic A. fumigatus extracts to inhibit specific IgE during a 40-day incubation period at $37^{\circ} \mathrm{C}$ was evaluated using a specific $\mathrm{IgE}$ inhibition ELISA (Fig. 4). We clearly demonstrate that specific IgE inhibition in fresh extract was dramatically higher compared to non-treated extracts which had decreased to $27 \%$. Treating allergenic $A$. fumigatus extracts with a stabilizer that contained Arg/Glu or Lys/Glu led to a higher specific IgE inhibition compared to the nontreated extract, but similar to fresh extract. Furthermore, we found that the specific IgE inhibition for $\mathrm{Arg} / \mathrm{Glu}$ 
and Lys/Glu treatments were $96 \%$ and $92 \%$ respectively. Although there was no significant difference between these two treatments, Arg/Glu treatment was more favorable. Interestingly, we found similar results with allergenic $C$. herbarum extracts (Fig. 5). Overall, our results show that there is a significant difference between fresh extract and non- treated extracts. While non-treated extracts experienced $28 \%$ allergenic reactivity and inhibition, Arg/Glu treatment, consequently, protected $97 \%$ of allergenic mold activity. Lastly, extracts treated with the stabilizer formulation containing Lys/Glu preserved $88 \%$ of allergenic mold activity after 40 days, which is significantly different from using Arg/Glu.

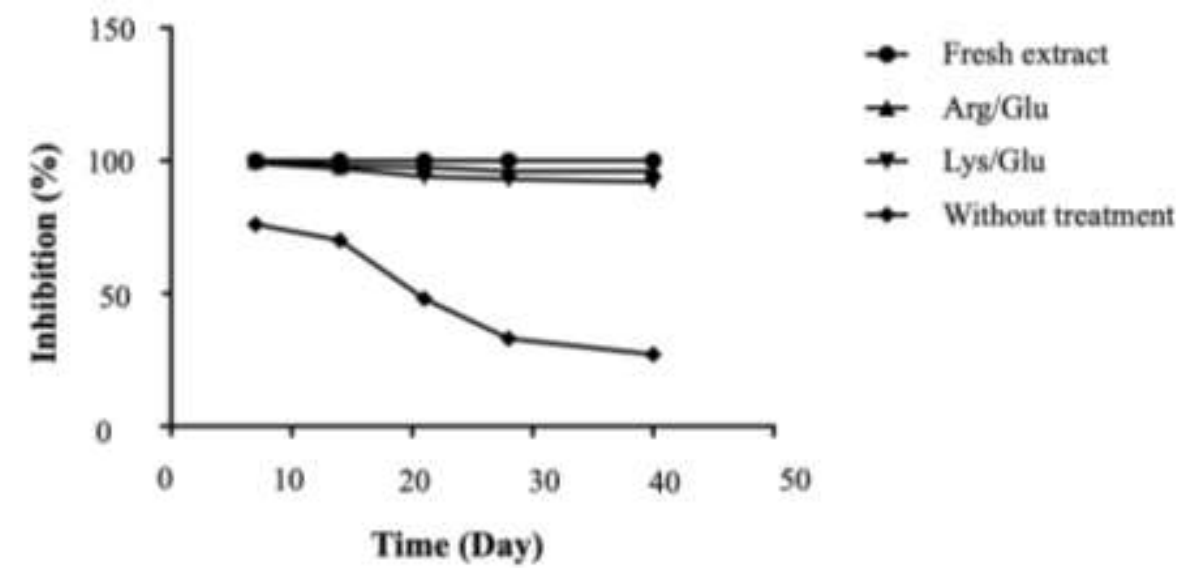

Fig. 4. Specific IgE inhibition with Aspergillus allergenic extract, which treated with different stabilizers during 40 days of incubation at $37^{\circ} \mathrm{C}$.

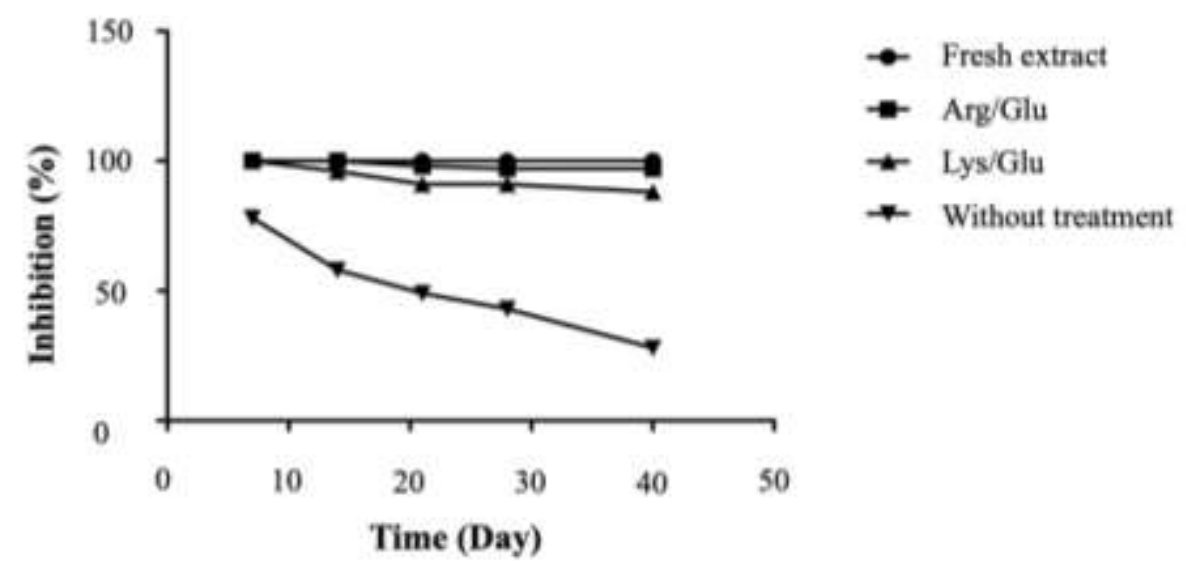

Fig. 5. Specific IgE inhibition with Cladosporium allergenic extract with different stabilizers during 40 days of incubation at $37^{\circ} \mathrm{C}$.

\section{Discussion}

With the rise in allergic diseases, allergenic extracts must remain stable and therefore, active during longterm storage conditions for their continued use in clinical and research settings. Allergenic extract stability greatly depends on the number and type of allergen present. Indeed, the presence of stabilizers and another chemical material may also play a critical role in preserving stability (12). Accordingly, any given extract may lose allergenic capacity due to the degradation of specific allergens rather than a general reduction in the potency of all allergens over time (16). Therefore, specific factors affecting stability must be clearly identified to ensure the functional longevity of allergenic extracts. Furthermore, a universal stabilizer formulation cannot prolong the potency of an entire extract containing allergens of diverse origin such as house dust mites, animalrelated allergens, molds or pollens (12). Hence, this study was an attempt to introduce a specific stabilizing formulation for allergenic molds. 
Different extraction methods can profoundly affect the potency of allergenic mold extracts. However, to gain a critical understanding of the factors affecting the stability and potency of mold extracts, high-quality extracts are required. In this study, we used a high yield extraction protocol that included an anti-protease supplement to increase the quantity and potency of our allergen of interest. Further, since molds are heavily pigmented with melanin, we included PVP during the extraction process to prevent protein precipitates. Moreover, PVP also binds to polyphenols, which can greatly improve yield. Additionally, EDTA, PMSF, and sodium chloride $(\mathrm{NaCl})$ were also added to inhibit protease activity $(17,18)$.

Most pharmaceutical companies suggest an expiry date of two years for skin prick tests(19). According to the Arrhenius equation, we increased the temperature to $37{ }^{\circ} \mathrm{C}$ and achieved an expiration date of 28 days, which is equivalent to two years at $4{ }^{\circ} \mathrm{C}$ (15). Eventually, a 40-day incubation period at $37{ }^{\circ} \mathrm{C}$ was used to determine the most effective stabilizer formulation after 28 days. Similar to our experimental system, others have also evaluated extract stability at incubations different from two years at $4{ }^{\circ} \mathrm{C}$, such as 3 days of incubation at $56^{\circ} \mathrm{C}(20)$. Moreover, additional days including day $7,14,21$, and 28 were evaluated to follow an IgE-mediated allergenic activity trend and to reveal any possible points of degradation. Overall, we extended the incubation period to reveal the stabilizing potency of all stabilizers used in this study.

Most articles recommend using 50\% glycerol, which has been suggested to be a good stabilizer as it is a common a preservative found in various skin prick tests (10). According to our results, $50 \%$ glycerol on its own was unable to stabilize allergenic activity during our specified incubation period and temperature. Since glycerol is a strong irritant, we used $20 \%$ glycerol. At this concentration, we aimed to maintain a similar stability as claimed by $50 \%$ glycerol users, while preventing bacterial growth (11). Though 20\% glycerol was not sufficient to maintain allergenic activity alone, formulations containing $20 \%$ glycerol with other components demonstrated better stability. According to recent evidence, allergenic pollen extracts without glycerol, but had included amino acids, had higher stability compared to glycerol-based extracts that contained $50 \%$ glycerol (20). In addition to glycerol, others have shown that the addition of $\beta$-Ala or $\mathrm{h} \mathrm{Alb}$ to the final formulation can also stabilize allergenic potency (21). However, we showed that $\beta$-Ala or h Alb individually was unable to maintain allergenic activity during a 28-day incubation period at $37^{\circ} \mathrm{C}$.

Some studies have reported that sugars and polyols can improve stability of protein-based media (22). In our study, we found that sucrose and sorbitol were only effective at an equal concentration to proteins. Further, there was a dramatic decline in allergenic activity if sorbitol was reduced to $25 \%$. Therefore, reducing sorbitol would not be applicable to our purpose, because we found stability to be less than or similar to extracts containing no sugar.

Though there is an overwhelming body of evidence to illustrate the importance of amino acids in stabilizing protein-based media, but their role in the allergenic extracts is much less characterized in the literature $(23,24)$ Therefore, both Arg and Glu were evaluated in our extract separately and in combination with each other. Separately, Arg and Glu was able to preserve allergenic activity until day 28 but not after 40 days at $37^{\circ} \mathrm{C}$. Thus, the individual use of Arg or Glu is not sufficient to protecting thermostability and allergenic activity of mold extracts during our desired incubation conditions.

When used together, Arg and Glu improved stability remarkably and maintained allergenic activity until day 40. Such a drastic observation could have occurred because Arg or Glu are both charged amino acids, and using them individually could have a profound or permanent effect on the net charge of proteins which could, therefore, promote degradation processes (14).

In addition, based on our observations of the allergenic potency of Alt al and other molds with our selected and altered formulation, we concluded that replacing Arg with Lys is not necessary to improve potency of mold extracts. It is unlike previous study on pollen allergenic extracts(23).

As this particular area of study is not well characterized, there are many potential opportunities for future investigations and immunotherapies, as there a plethora of proteins and glycoproteins within mold extracts that also require our attention $(25,26)$. There is conflicting consensus within the literature on what exactly determines the best possible outcome for allergenic formulations, in terms of stability, for instance, some studies suggest using other amino acids (27), while others recommend other sugars such as 
mannitol and trehalose to stabilize extracts (28). In addition, ions used in buffers may also play a pivotal role in stabilizing solutions, and preventing protein degradation (10).

Though there is a critical need for examining all fungi and other chemical stabilizers, this paper provides a simple formulation to improve allergenic fungi extract stability, since many industrial formulations are strictly for commercial use, and thus, confidential property.

In addition to using sorbitol, sucrose, and glycerol, which are among the most traditionally used preservatives to date, we found that amino acids play a larger role in stabilizing allergenic activity in mold

\section{References}

1. Rapiejko P, Jurkiewicz D, Pietruszewska W, Zielnik-Jurkiewicz B, Woron J, Lipiec A. Treatment strategy of allergic rhinitis in the face of modern world threats. Otolaryngol Pol. 2018;72(2):1-12.

2. Seidman MD, Gurgel RK, Lin SY, Schwartz SR, Baroody FM, Bonner JR, et al. Clinical practice guideline: allergic rhinitis. Otolaryngology Head Neck Surg. 2015;152(1 suppl):S1-43.

3. Cardona V, Demoly P, Dreborg S, Kalpaklioglu AF, Klimek L, Muraro A, et al. Current practice of allergy diagnosis and the potential impact of regulation in Europe. Allergy. 2018;73(2):323-327.

4. Kołodziejczyk K, Bożek A, Jarząb J, Gawlik R. The clinical differences of asthma in patients with molds allergy. Pneumonol Alergol Pol. 2016;84(2):81-6.

5. Fukutomi Y, Taniguchi M. Sensitization to fungal allergens: resolved and unresolved issues. Allergol Int. 2015;64(4):321-31.

6. Twaroch TE, Curin M, Valenta R, Swoboda I. Mold allergens in respiratory allergy: from structure to therapy. Allergy Asthma Immunol Res. 2015;7(3):205-20.

7. Cox L, Nelson H, Lockey R. Allergen immunotherapy: a practice parameter third update. $\mathrm{J}$ Allergy Clin Immunol. 2011;127(1 suppl):S1-55.

8. Raulf M, Quirce S, Vandenplas O. Addressing molecular diagnosis of occupational allergies. Curr Allergy Asthma Rep. 2018;18(1):6.

9. Hamilton RG. Assessment of human allergic diseases. Clinical Immunology. 2019:1283-95.

10. Ariaee N, Varasteh A, Jabbari Azad F, Sankian M.

Preparation allergenic pollen extracts; the points should extracts. Though we proved that Arg/Glu was the best candidate during our study, this simple formulation should not be generalized for all allergenic extracts since their allergen content may vary in type and number. In this regard, future research should be conducted for other extracts and amino acids.

\section{Acknowledgment}

The authors wish to thank Dr. Hossein Zarinfar (Ph. D. in Mycology) for his sincere collaboration. This study was performed under financial support of Research Deputy of Mashhad University of Medical Sciences (grant number: 940043).

be considered to make high-quality products. J Immunoassay Immunochem. 2018:40(1):26-39.

11. Plunkett G. Update: stability of allergen extracts to establish expiration dating. Curr Opin Otolaryngol Head Neck Surg. 2016;24(3):261-9.

12. Grier TJ, Hall DM, Duncan EA, Gada SM. Allergen stabilities and compatibilities in immunotherapy mixtures that contain cat, dog, dust mite, and cockroach extracts. Ann Allergy Asthma Immunol. 2015;115(6):496-502.

13. Oskouei YM, Hosseini RF, Ahanchian H, Jarahi L, Ariaee N, Azad FJ. Report of common aeroallergens among allergic patients in northeastern Iran. Iran J Otorhinolaryngol. 2017;29(91):89-94.

14. Golovanov AP, Hautbergue GM, Wilson SA, Lian LY. A simple method for improving protein solubility and long-term stability. J Am Chem Soc. 2004;126(29):8933-9.

15. Pawar S, Dell AI, Savage VM, Knies JL. Real versus artificial variation in the thermal sensitivity of biological traits. The American Naturalist. 2016;187(2): E41-52.

16. Zhuang Y, Dreskin SC. Redefining the major peanut allergens. Immunol Res. 2013;55(1-3):125-34.

17. Dellavalle PD, Cabrera A, Alem D, Larrañaga P, Ferreira F, Dalla Rizza M. Antifungal activity of medicinal plant extracts against phytopathogenic fungus Alternaria spp. Chilean journal of agricultural research. 2011;71(2):231-239.

18. Paris S, Fitting C, Ramirez E, Latge JP, David B. Comparison of different extraction methods of Alternaria allergens. Journal of allergy and clinical immunology. 1990;85(5):941-8. 
19. Ariaee N, Varasteh A, Jabbari Azad F, Sankian M. Preparation allergenic pollen extracts; the points should be considered to make high-quality products. $\mathrm{J}$ Immunoassay Immunochem. 2019;40(1):26-39.

20. Mansouritorghabeh H, Sankian M, Varasteh AR, Moghadam M, Khameneh B, Farid-Hosseini R, et al. Determination of Optimum Excipients for Platanus orientalis Pollen Extract by Accelerating Chemical Stability Test and Their Synergistic Effect. Reports of Biochemistry and Molecular Biology. 2019;7(2):189-195.

21. Plunkett G. Stability of allergen extracts used in skin testing and immunotherapy. Curr Opin Otolaryngol Head Neck Surg. 2008;16(3):285-91.

22. Bijli K, Singh B, Sridhara S, Gaur S, Arora N. Effect of various stabilizing agents on Imperata cylindrica grass pollen allergen extract. Clin Exp Allergy. 2003;33(1):65-71.

23. Ariaee N, Sankian M, Varasteh A, Hosseinpour M, Jabbari F. Determining if Amino Acids Can Affect Stabilizing Allergenic Pollen Extracts.
Reports of Biochemistry \& Molecular Biology. 2020;8(4):394-400.

24. Platts L, Darby SJ, Falconer RJ. Control of Globular Protein Thermal Stability in Aqueous Formulations by the Positively Charged Amino Acid Excipients. J Pharm Sci. 2016;105(12):3532-3536.

25 . Esch RE. Manufacturing and standardizing fungal allergen products. J Allergy Clin Immunol. 2004;113(2):210-5.

26. Gasilova N, Girault HH. Bioanalytical methods for food allergy diagnosis, allergen detection and new allergen discovery. Bioanalysis. 2015;7(9):1175-90.

27. Hoseini RF, Jabbari F, Rezaee A, Rafatpanah $\mathrm{H}$, Yousefzadeh $\mathrm{H}$, Ariaee N, et al. House dust mite sublingual-swallow immunotherapy in perennial rhinitis: a double-blind, placebocontrolled Iranian study. J Biol Regul Homeost Agents. 2018;32(1):83-88.

28. Zimmer J, Vieths S, Kaul S. Standardization and regulation of allergen products in the European Union. Curr Allergy Asthma Rep. 2016;16(3):21. 